\title{
Seismological insights into solar and stellar magnetic activity cycles
}

\author{
Anne-Marie Broomhall ${ }^{1,2, \star}$ \\ ${ }^{1}$ Institute of Advanced Studies, University of Warwick, Coventry, CV4 7HS, UK \\ ${ }^{2}$ Centre for Fusion, Space, and Astrophysics, Department of Physics, University of Warwick, Coventry CV4 7AL, UK
}

\begin{abstract}
The Sun's magnetic activity cycle varies primarily on a time scale of approximately $11 \mathrm{yrs}$ from minimum to maximum and back again. It is well-known that the properties of the Sun's acoustic oscillations are affected by the near-surface internal magnetic field: Frequencies, damping rates, and powers are all known to vary systematically with solar cycle. Careful observation of these variations, therefore, allows aspects of the Sun's internal magnetic field to be inferred. However, the Sun is just one star and, with CoROT and Kepler in particular, oscillations can now be observed for thousands of other stars. However, despite many stars showing signs of magnetic activity in their lightcurves, to date, activity cycle-like variations in the properties of asteroseimic oscillations are sparse. Nevertheless, studying the solar-stellar connection advances our understanding of solar and stellar magnetic fields. For example, placing our Sun's activity cycle in a stellar context suggests that the Sun may be unusual. Conversely, observations of quasi-periodic pulsations in stellar flares suggest the underlying physics of solar and stellar flares are the same.
\end{abstract}

\section{Introduction}

The Sun's activity cycle varies primarily on a timescale of 11 years. However, each cycle is different. Indeed the last solar minimum was generally regarded as being unusually long and deep, while solar cycle 24 is the weakest seen since the beginning of the $20^{\text {th }}$ century. One of the most obvious manifestations of solar activity on the solar surface is sunspots and the number of sunspots is known to track the solar cycle. Sunspot records extend back hundreds of years and records show that both the length and the amplitude of sunspot cycles vary from one cycle to the next, with lengths observed in the range of approximately 9-14yrs. There is also evidence that the amplitudes of the cycles themselves vary systematically over what is know as a Gleissburg cycle, which has a periodicity of about 70$100 y$ rs, or around 9 cycles. The recent cycles are generally referred to as the Modern Maxima because, although the current cycle is small, that is just in comparison to recent cycles. There are also Grand Minima, the most famous of which is the Maunder Minimum, where almost no sunspots were observed for an extended period of time. Reconstructions based on radioisotopes of tree rings and icecores indicate other grand minima and maxima existed. These reconstructions also reveal a much longer periodicity of about $210 y$ rs, which is known as the Seuss cycle.

There are also periodic variations associated with magnetic activity that have characteristic timescales shorter than $11 \mathrm{yrs}$. Those shorter than 1yr are collectively referred to as Rieger-like periodicities [1]. While there is also strong evidence for what is loosely referred to as the quasi-

\footnotetext{
^e-mail: a-m.broomhall@ warwick.ac.uk
}

biennial oscillation (QBO), although this term is generally attributed to any periodicity in the range 1-4yr. Both the Rieger and the quasi-biennial signal are fairly ubiquitous, being observed in activity proxies sensitive to the solar interior right out to the interplanetary medium [see 2, for a recent reviews]. The amplitude of the QBO varies as a function of time and is at a maximum at solar maximum and this feature is well-known and can be observed in all the vast majority activity proxies associated with the QBO.

To summarize the Sun's magnetic activity cycle is not just a simple $11 \mathrm{yr}$ variation but is far more complex. This paper describes helioseismic observations of the Sun's activity cycle (Section 2) and places these observations in a stellar context, discussing observations of stellar activity cycles and the potential for asteroseismology to reveal insights into the Sun's magnetic dynamo (Section 3). We finish, in Section 4, with a slight change of pace, switching to from stellar interiors to stellar atmospheres, and describing how stellar flare seismology can link magnetic features of the Sun and stars.

\section{The helioseismic magnetic activity cycle}

Properties of the Sun's acoustic (p mode) oscillations vary through the solar cycle. Figure 1 shows comparison of the profiles fitted to a p-mode spectrum at activity maximum and activity minimum. It is clear to see that the frequencies of the oscillations vary with time, as do the heights of the peaks. Less visible are variations in the widths of the profiles, which increase between cycle minimum and cycle maximum. We now describe the solar cycle varia- 
tions in these parameters in more detail, beginning with the mode frequencies.
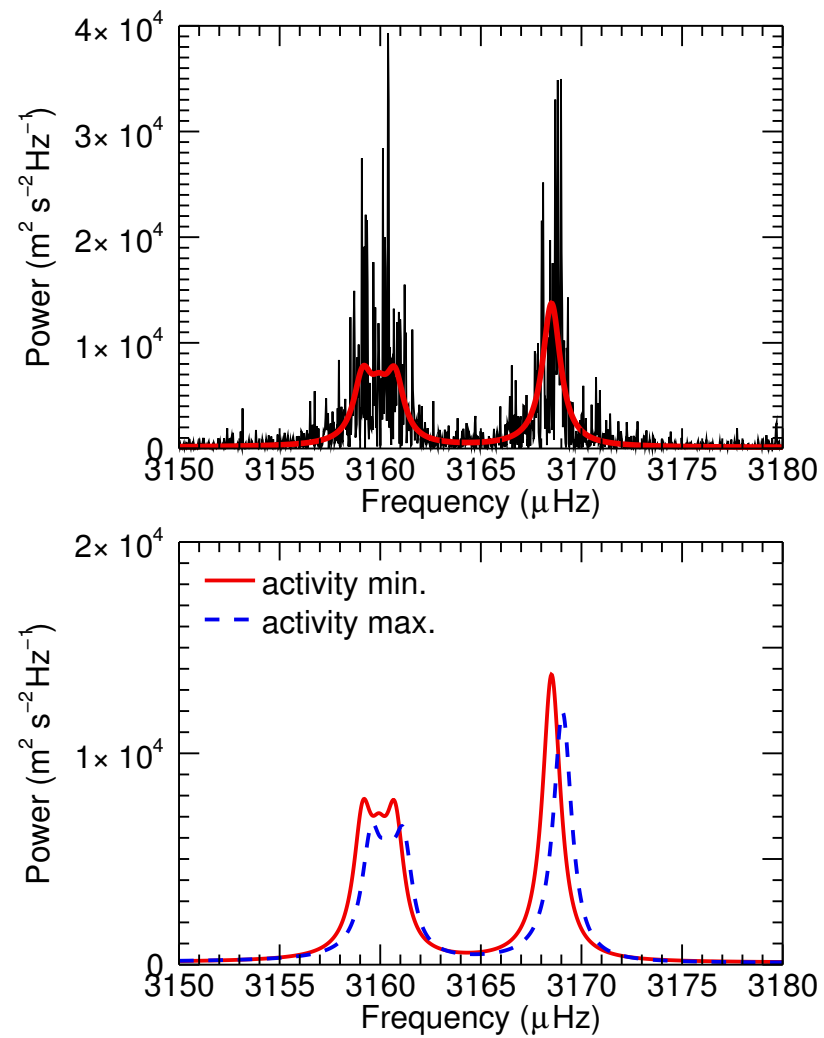

Figure 1: Top: Portion of a Sun-as-a-star power spectrum concentrating on an $\ell=2, \ell=0$ pair. Black fine line shows the data observed by Birmingham Solar Oscillations Network. Thick red line shows the asymmetric model fitted to the data. Bottom: A comparison of the asymmetric Lorentzian models fitted to an $\ell=0, \ell=2$ pair at solar maximum and solar minimum. The solid red line shows activity minimum. This is the same model fit as shown in the top panel. The blue dashed line shows a fit to data observed at activity maximum.

\subsection{Solar cycle variations in $p$ mode frequencies}

There are two ways in which we generally determine the change in frequencies as a function of time and these methods have been employed to study variations in both solar and stellar p-mode frequencies. The first method determines the change in frequency directly from the fitted mode frequencies. Let $\delta v_{\ell, n}(t)$ be the change in frequency, or "frequency shift", as a function of time. The frequency shift is then given by

$$
\delta v_{\ell, n}(t)=v_{\ell, n}(t)-\overline{v_{\ell, n}},
$$

where $v_{\ell, n}(t)$ is the frequency of a mode at time $t$ and $\overline{v_{\ell, n}}$ is the "reference frequency" for that mode. This reference frequency is usually obtained by determining the average frequency of a the centroid of an individual mode over a longer period of time, say at solar minimum or the entire epoch under consideration. Although technically one can then consider frequency shifts for each individual mode, more frequently we determine averages over say a particular range of $n$ and/or $\ell$.

The second method determines the cross-correlation of time-lagged power spectra. To determine the frequency shift it is necessary to fit a function to the cross-correlation, where the average frequency shift is given by the frequency offset of the peak about zero. This method determines the average frequency shift for all modes in the frequency range for which the cross-correlation was determined. Therefore, it is possible to control the frequency range under consideration. However, it is not possible to isolate particular $\ell$ when using Sun-as-a-star data.

Chaplin et al. [3] compared the two methods for studying the solar cycle and found that the obtained frequency shifts were, for the most part, in very good agreement. However, they determined that the uncertainties associated with the cross-correlation method were systematically larger than those associated with the the frequency shifts obtained from the individual mode frequencies. In reality the decision over which method to utilise will depend on the quality and quantity of data available: The cross-correlation method may be a better option when considering low signal-to-noise data or short data series, such as those utilised in asteroseismic analyses, from which it is difficult to robustly fit profiles to the individual mode peaks.

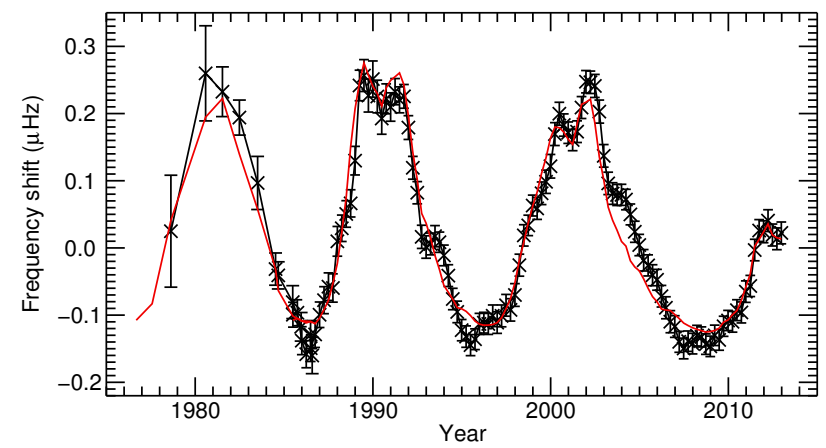

Figure 2: Average frequency shift of Sun-as-a-star oscillations as a function of time (black data points). The frequency shifts were obtained from Birmingham Solar Oscillations Network (BiSON) data. Frequency shifts were determined using Equation 1. Overplotted in red is a scaled and shifted version of the $10.7 \mathrm{~cm}$ flux, included for comparison purposes.

Figure 2 shows the average change in seismic frequency as a function of time. The frequency shifts were obtained using the individual mode frequencies (the first method described above). The frequencies were determined by fitting profiles, like those shown in Figure 1, to frequency-power spectra created from time-shifted $365 \mathrm{~d}$ time series observed by the Birmingham Solar Oscillations Network (BiSON). BiSON makes unresolved (Sunas-a-star) Doppler velocity observations of the Sun. As such the BiSON data are only sensitive to those oscillations with the largest horizontal scales (or the lowest harmonic degrees, $\ell \leq 3$ ). The $11 \mathrm{yr}$ cycle is clear visible. The frequency shift, and therefore the mode frequencies, are at a maximum at cycle maximum, with modes at about 
$3000 \mu \mathrm{Hz}$ experiencing a shift of approximately $0.4 \mu \mathrm{Hz}$ between cycle minimum and cycle maximum.

The exact causes of the frequency shifts are still somewhat uncertain. Broadly speaking there are both direct and indirect effects. The direct effects involve the Lorentz force, which provides an additional restoring force. While the indirect effects involve changes to the properties of the cavity in which the modes are trapped. For example, the presence of a magnetic field may alter the size of the acoustic cavity. The exact balance between direct and indirect effects is not clear. For example, Dziembowski \& Goode [4] believe that the indirect effects are predominantly responsible for the frequency shifts. However, they do find that there is a frequency dependence, with the direct effect playing a more important role in shifting lowfrequency modes. More recently, Santos et al., [5] find that approximately $30 \%$ of the frequency shifts can be accounted for by sunspots. We note that this proportion is dependent on the inclusion of a smooth component that represents long-term cycle variations.
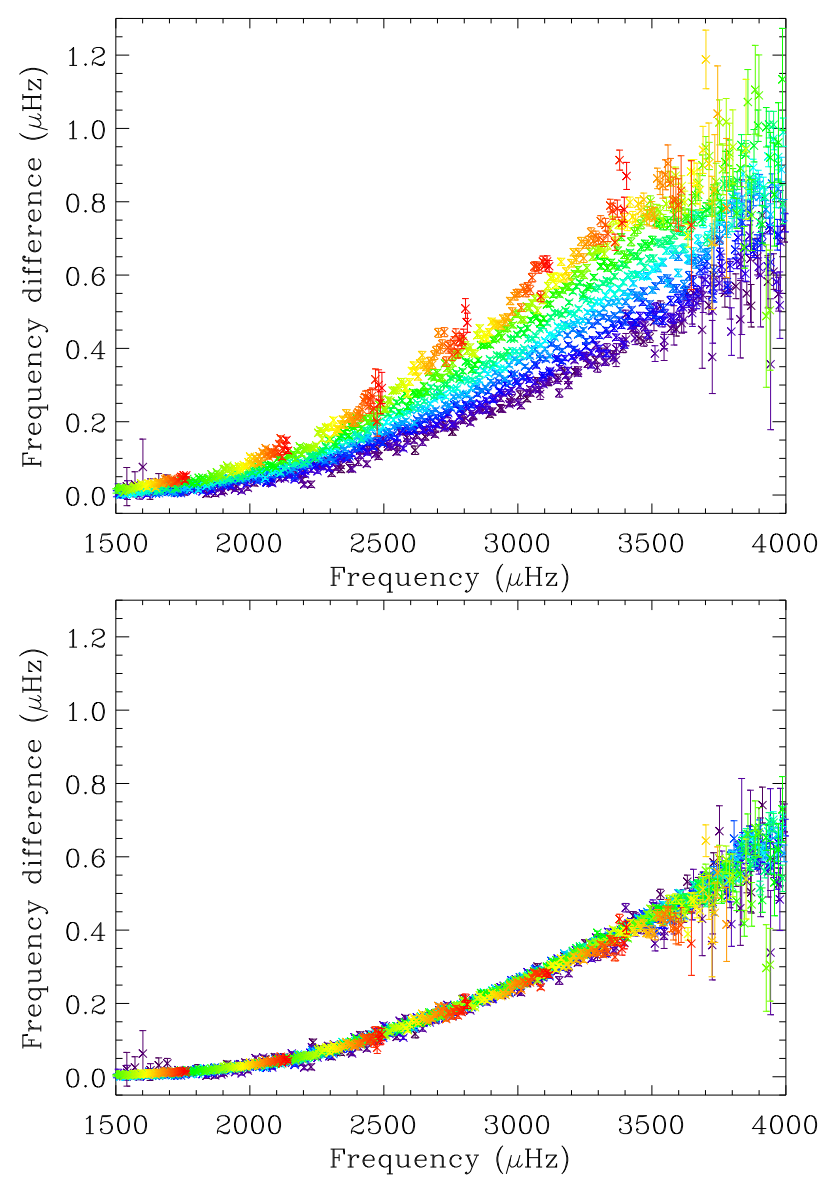

Figure 3: Shift in frequency observed between cycle minimum and cycle maximum as a function of mode frequency. The frequencies are obtained from the standard Global Oscillations Network Group (GONG) pipeline. The colours indicate the degree of the mode, with purple representing the lowest degree modes and red representing the highest degree modes. Figures taken from [6].
The top panel in Figure 3 shows the change in frequency between cycle minimum and cycle maximum as a function of frequency. It is clear that the size of the frequency shift depends both on the oscillation frequency and on the harmonic degree of the mode [also see e.g. 7-12]. The harmonic degree tells us about how deep in the solar interior the oscillations travel, with low degree modes travelling deeper in the solar interior than high degree modes. The degree dependence can be removed by normalizing by mode inertia [e.g. 11]. The normalized mode inertia is defined by Christensen-Dalsgaard [13] as

$$
\begin{aligned}
I_{n, l} & =M_{\odot}^{-1} \int_{v}|\xi|^{2} \rho \mathrm{d} V \\
& =4 \pi M_{\odot}^{-1} \int_{0}^{R_{s}}|\xi|^{2} \rho r^{2} \mathrm{~d} r \\
& =\frac{M_{n, l}}{M_{\odot}}
\end{aligned}
$$

where $\xi$ is the displacement associated with a mode, suitably normalized at the photosphere, $V$ is the volume of the Sun, and $M_{\odot}$ is the mass of the Sun. $M_{n, l}$ is the 'mass' associated with a mode. The bottom panel of Figure 3 shows that the mode inertia normalization leaves a functional form that is dependent on frequency alone. The fact that the inertia-corrected frequency shifts show a strong frequency dependence but little or no dependence on degree implies that most of frequency changes are caused by effects confined to near-surface layers, specifically the upper few $100 \mathrm{~km}$ of the solar interior [e.g. 7, 14]. In fact, latitudinal inversions show that the frequency shifts are highly correlated with the observed surface magnetic field and even manage to replicate the well-known butterfly diagram [e.g. 15, 16].

In Sun-as-a-star data there is an additional dependence that occurs because of the latitudinal distribution of the magnetic field, as shown in Figure 4. The sectoral $|m|=\ell$ components are more sensitive to low latitudes than the zonal $m=0$ components. Since the magnetic activity associated with the $11 \mathrm{yr}$ solar cycle is also concentrated near the solar equator this means that the frequencies of the sectoral components are shifted by a larger amount than zonal components [17]. This is important for Sun-as-a-star observations since the frequencies obtained for a $\ell=2$ mode are dominated by the sectoral components as these are by far the most prominent components of an $\ell=2$ mode observed in a frequency-power spectrum. More specifically, for an $\ell=2$ mode $90 \%$ of the final frequency comes from the sectoral component and only $10 \%$ from the zonal component. Consequently, in Sun-as-a-star data, $\ell=2$ modes experience a larger shift in frequency than $\ell=0$ modes of approximately the same frequency. This could become important in asteroseismic studies of stellar activity cycles as it any degree dependence on the size of the shift can then be used to infer the latitudinal distribution of the star's activity.

\subsection{Quasi-biennial Oscillation}

Figure 5 shows the residuals of the frequency shifts once the $11 \mathrm{yr}$ variation is removed. This was done by subtract- 


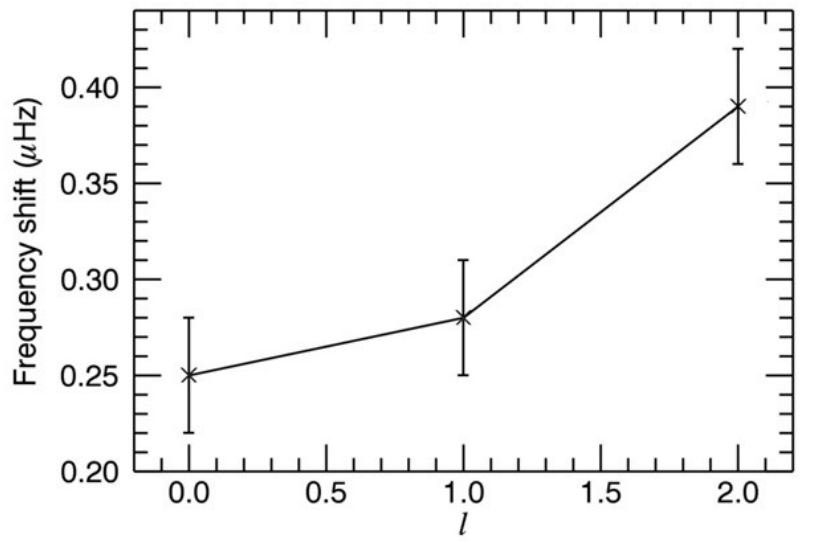

Figure 4: Frequency shift as a function of degree as seen in Sunas-a-star data.

ing a smoothed component, where a boxcar smoothing of $3 \mathrm{yr}$ was performed. Variations on timescales shorter than $11 \mathrm{yr}$ are clearly visible. These variations are commonly referred to as a quasi-biennial oscillation (QBO) and Kolotkov et al., [18] demonstrated that the helioseismic QBO has an average periodicity of approximately $2.6 \mathrm{yr}$. The quasi-biennial variations seen in the frequency shifts are highly correlated with those seen sunspot number, and this is true for a large number of other activity proxies [19]. Furthermore, the QBO observed in different activity proxies even have the same amplitude relative to the $11 \mathrm{yr}$ variation. Another feature of the $\mathrm{QBO}$ common to helioseismic frequency shifts and other measures of activity is the observed amplitude variation, such that the amplitude of the QBO is at a maximum at solar maximum. However, mystery still surrounds the QBO: Whether or not it is a real phenomenon or an artifact of the data analysis techniques used is still debated, as is the potential physical mechanism responsible for the QBO. Current theories include a second, near-surface dynamo [20], the instability of magnetic Rossby waves in the solar tachocline [21], spatiotemporal fragmentation [22] and beating between dipole and quadropole magnetic configurations of a dynamo [23, 24].

\subsection{Cycle-to-cycle variations}

Although we don't have enough helioseismic data to look at the longer-term cycles mentioned in Section 1, we have been able to show that the cycles are different. Figure 6 shows the frequency shifts that have been obtained by averaging over 3 different frequency ranges. Notice that the $10.7 \mathrm{~cm}$ flux and sunspot number diverge in cycle 23 , as is observed by, for example, Livingston, Penn, \& Svalgaard [25]. The frequency shifts also appear to diverge during cycle 23 in all frequency ranges. Perhaps most eyecatching, though, is the behaviour of the low-frequency modes in cycle 23 . These low-frequency modes appear to show a significant shift in cycle 22 but almost no solar-cycle shift in cycle 23.

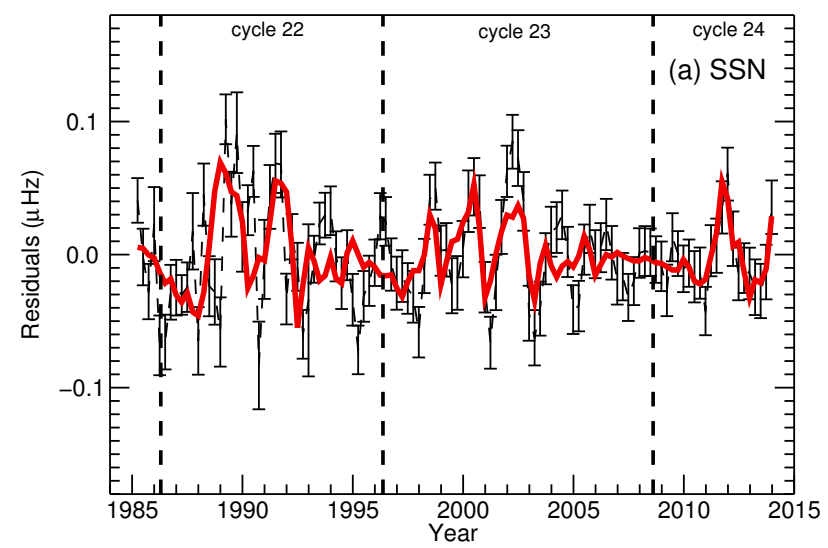

Figure 5: Residuals of frequency shifts once $11 \mathrm{yr}$ cycle has been removed (black data points, with dashed line). Overplotted in the red solid line is a scaled version of the sunspot number residuals. We note that the scaling has been made with respect to the $11 \mathrm{yr}$ cycle.

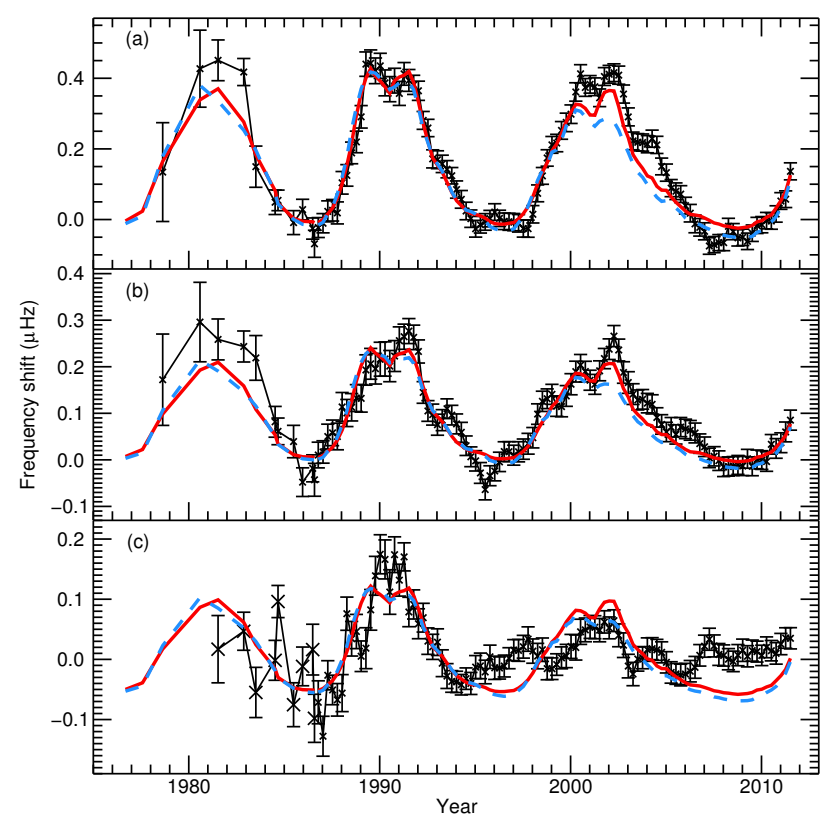

Figure 6: Average Sun-as-a-star frequency shifts as a function of time. Panel (a): Frequency shifts seen in modes with frequencies in the range $2920<v \leq 3450 \mu \mathrm{Hz}$. Panel (b): Frequency shifts seen in modes with frequencies in the range $2400<v \leq 2920 \mu \mathrm{Hz}$. Panel (c): Frequency shifts seen in modes with frequencies in the range $1860<v \leq 2400 \mu \mathrm{Hz}$. Only modes with degrees in the range $0 \leq \ell \leq 2$ were considered. In all panels the red solid line represents a scaled and shifted version of the $10.7 \mathrm{~cm}$ flux and the blue line dashed line represents a scaled and shifted version of the sunspot number. Taken from [26].

The upper turning point of modes varies with frequency, with high-frequency modes turning at greater radii than low-frequency modes. So one possible explanation for the effect is that the magnetic layer responsible for the solar cycle shift has become thinner: In cycle 22 the magnetic layer is thick enough that the upper turning points of both the low and high frequency ranges lie within the 
magnetic layer. However, in cycle 23 this layer is thinner so that the upper turning point of the high-frequency modes still lie within this layer but the upper turning point of the low frequency modes lies underneath the layer. An alternative explanation is that the cavity properties have changed, altering the upper turning point of the modes. To isolate the region responsible we can look at which parts of the Sun the modes are sensitive to using kernals. It can be shown that the changes between cycle 22 and 23 must occur above $2400 \mathrm{~km}$ [26]. Salabert et al. [27] showed a similar result can be obtained using GOLF data.

\subsection{Solar cycle variations in oscillation powers and damping rates}

In addition to the mode frequencies, the damping rates and powers of the oscillations also show solar cycle variations. The damping rates are at a maximum at solar maximum while the powers are at a minimum [28]. The in-phase behaviour of the damping rates and anti-phase behaviour of the mode powers could be useful when searching for activity cycles in asteroseismic data, as any similar behaviour would add weight to any claimed detections. We note that the fractional variations in the mode widths appear to show double maxima, which are associated with the QBO. It is well known that active regions suppress mode powers and affect mode lifetimes but the mechanisms are still poorly understood [e.g. 29, 30]. We note that Houdek et al. [31] demonstrated that changes in the convection can affect mode damping rates and theorized that such changes could be brought about through the presence of magnetic structures.

\section{Stellar activity cycles}

Evidence for stellar activity cycles has now been observed for some time, primarily because of the Mount Wilson Calcium HK survey [e.g. 32]. The calcium HK index is a measure of the activity in the chromosphere. The Mount Wilson survey showed that other stars exhibit activity cycles that are very reminiscent of our own Sun's. However, it also demonstrated that other stars show far more irregular activity-related variations, while others appear to show no variation whatsoever, with approximately $60 \%$ showing cyclic variability, $25 \%$ showing aperiodic variability, and $15 \%$ showing almost no variability (i.e. flat) [33].

Hopes were high that the long-term, high-precision asteroseismic observations of Kepler and CoROT might lead to observations of cycle-like variations in the oscillation parameters of other stars. The first evidence for such an asteroseismic activity cycle came from [34], who observed that the frequencies of the oscillations of HD49933, which was observed by CoROT, changed with time and that the amplitudes varied in anti-phase, as is observed for the solar data. Salabert et al. [35] examined the frequency dependence of the shifts, which increase with frequency. Again this is just as we see in the Sun. Salabert et al. [35] also noted that the shifts are larger than observed in the Sun. Observations of stellar activity induced frequency shifts
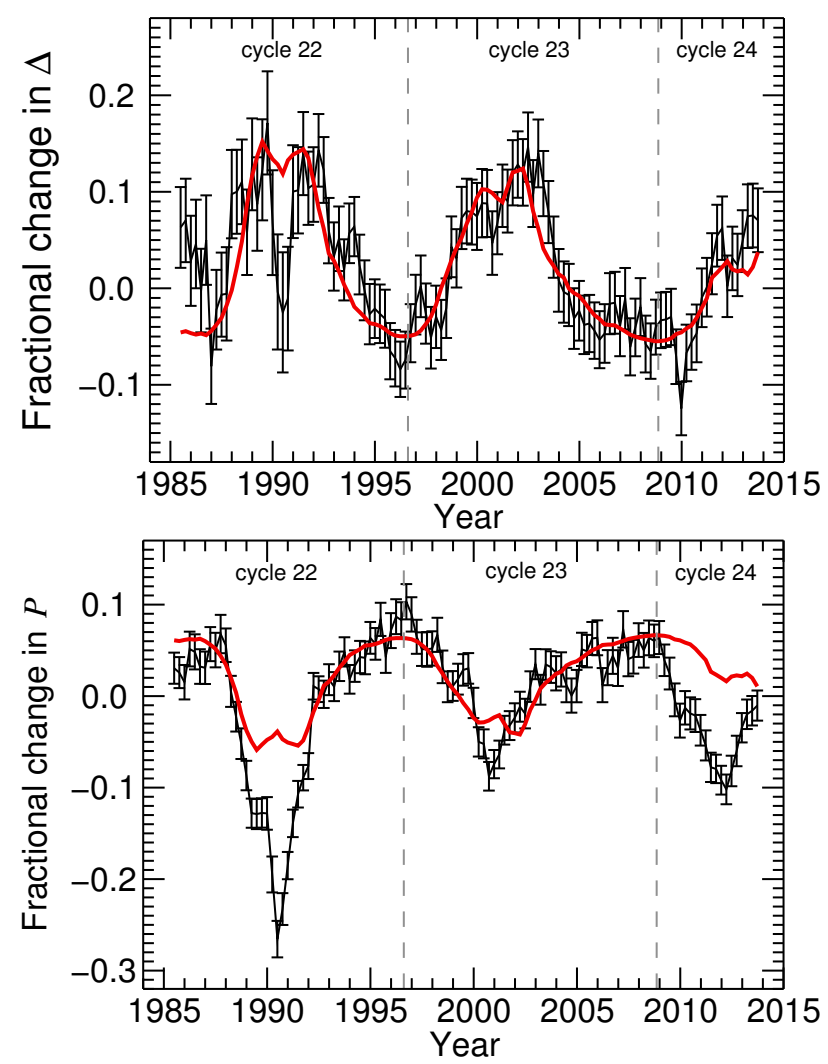

Figure 7: Top panel: Fractional variations in mode width as a function of time (black data points). Overplotted in red is a scaled and shifted version of the $10.7 \mathrm{~cm}$ flux for comparison. Bottom panel: Fractional variations in mode power as a function of time (black data points). Overplotted in red is an inversely scaled and shifted version of the $10.7 \mathrm{~cm}$ flux for comparison. The fractional variations in the mode widths and powers were averages over modes in the frequency range $2400 \leq v \leq 3500 \mu \mathrm{Hz}$. Mode parameters were obtained from Sun-as-a-star BiSON data and so only modes in the range $0 \leq \ell \leq 2$ were considered.

in different stars will place additional constraints on predictions over the size of shift one might expect [36, 37], which in turn will improve our understanding of the underlying causes of the freqeuncy shift (see discussion in Section 2.1).

Other examples of asteroseismic cycles however are rare. Salabert et al. [38] and Régulo et al. [39] are two recent papers looking for such asteroseismic cycles that do appear to detect systematic variations in $\mathrm{p}$ mode frequencies. Furthermore, these systematic variations appear to be in phase with measures of activity based on the variations in the light curves. However, the variations are still small, especially when compared to typical uncertainties. Kiefer et al.[40] also finds evidence for cycle-like variability in asteroseismic data. However, the question still arises as to why evidence for asteroseismic activity cycles is not more abundant.

Essentially there is a catch 22 situation: Ideally we want to look at stars that are reasonably active so the activity-induced frequency shifts are large enough to be statistically significant. Since, even in the best case sce- 
nario we only have approximately $4 \mathrm{yr}$ of Kepler data available we also need stars with shorter activity cycles than the Sun: As can be seen from Figure 8 there is a well known relation between a star's rotation rate and the length of its cycle: Very broadly, cycle length increases with increasing rotation rate. However, a more careful examination reveals that the cycle periods increase proportionally with rotation period along two distinctly different sequences, known as the active " $A$ " sequence and the inactive "I" sequence, with cooler and more slowly rotating stars generally lying on the I branch. Along each sequence the number of rotation periods per activity cycle is nearly the same, but the numbers are different for the different sequences. Bohm-Vitense [41] proposed that the branches are fed by different dynamo mechanisms with a near surface dynamo driving the A branch and a dynamo at the base of the convection zone responsible for the I branch. We can also see from Figure 8 that in terms of the primary cycles the younger stars generally lie on the A branch and the older stars like on the I branch.

There is also evidence that the faster a star rotates the more active it is: the X-ray luminosity ratio, $R_{X}=L_{X} / L_{\text {bol }}$, is proportional to Rossby number (Ro $=P_{\text {rot }} / \tau$, where $\tau$ is the convective turnover time at the base of the convection zone), until a saturation limit [see 42, and references therein]. The saturation limit occurs at Ro $=0.13 \pm 0.02$ and for Rossby numbers less than this value $R_{X}$ is constant and has a value of $\log R_{X}=-3.13 \pm 0.08$ [42]. Since Xrays are emitted from magnetically confined hot plasma in a star's corona an increase in $R_{X}$ is believed to correspond to an increase in activity. The higher the X-ray luminosity is the hotter or denser the corona is and so the more active the star is. This means that faster rotators are more active and are expected to have shorter activity cycles.

However, we know from observations of the Sun that magnetic field suppresses the amplitude of acoustic oscillations (see Section 2.4). This result is validated for other stars in [43], who demonstrated that the fraction of stars in which one can detect oscillations decreases as a function of increasing activity. Therefore, the faster a star rotates the shorter its activity cycle, but the more active the star is and the less likely we are to be able to detect acoustic oscillations. It is, therefore, perhaps understandable that more asteroseismic activity cycles have not been detected to date.

The Sun's primary $11 \mathrm{yr}$ cycle and secondary quasibiennial cycle have also been plotted in Figure 8. Neither match particularly well with the observations of the other stars and one proposed explanation for this is that the Sun is in transition. van Saders et al. [44] found a population of field stars in the Kepler field of view that were rotating faster than expected based on gyrochronology expectations. They explained this in terms of reduced magnetic braking efficiency beyond Ro $\sim 2$ i.e. the Sun's Rossby number. Metcalfe et al. [45] connect these results with chromospheric activity measures and conclude that the Sun's magnetic activity cycle really is unusual, being in an transitional state.
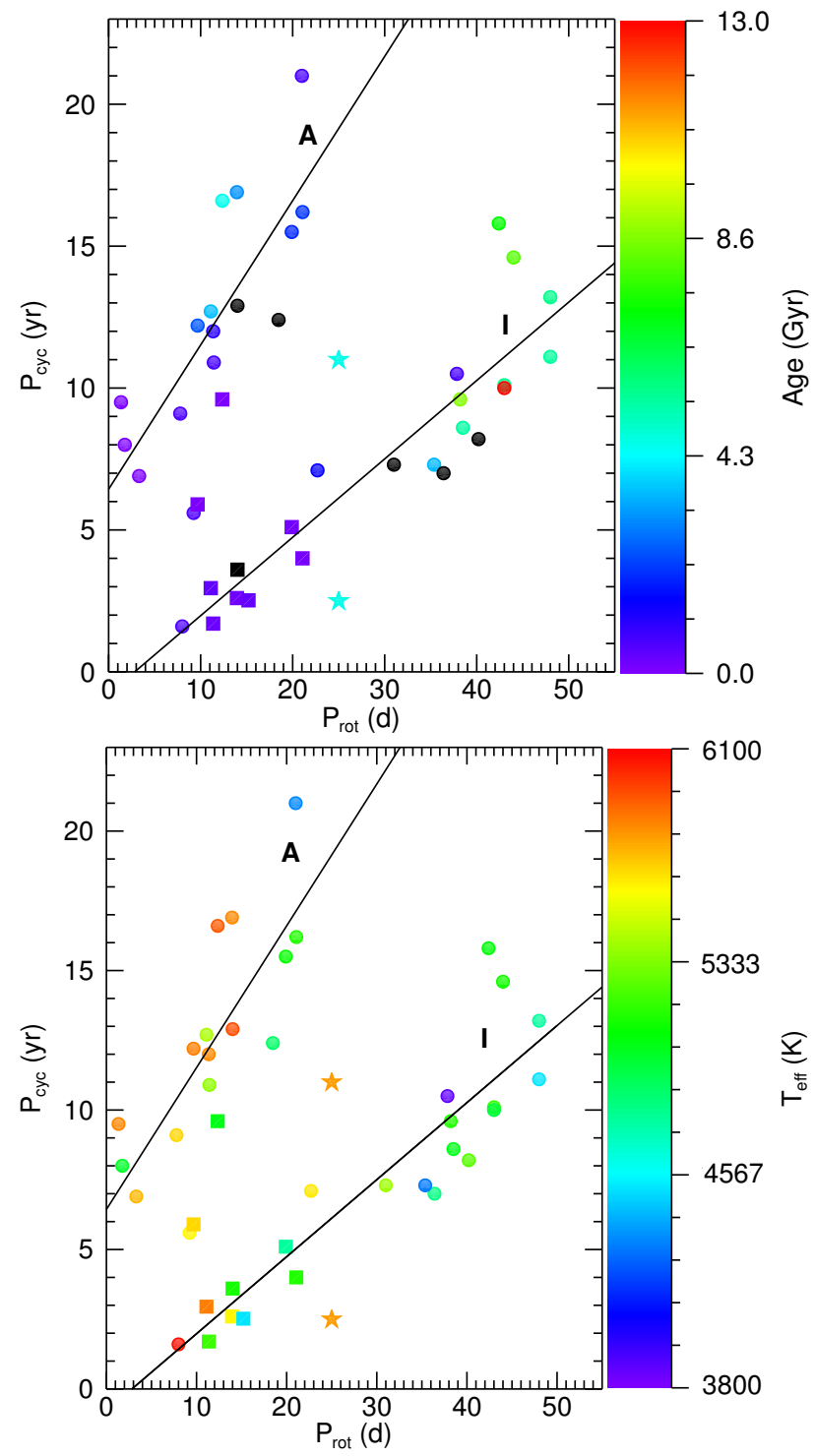

Figure 8: A Bohm-Vitense figure, adapted from [41], which plots cycle length as a function of a star's rotation rate. In the top panel colours indicate the age of the stars, where black indicates the age is as yet unknown. In the bottom panel colours indicate effective temperature. Circles represent primary cycles and squares represent secondary cycles. The two solid lines show best fits for the active "A" branch and the inactive "I" branch.

Finally, we recall from Section 1 that the variability in the Sun's magnetic activity is far more complex than even just the supposed primary $11 \mathrm{yr}$ and secondary quasibiennial cycles. Oláh et al. [46] re-examined the Mount Wilson HK survey and found multiple cycles in a large number of stars. Figure 9 is a Bohm-Vitense diagram constructed with the Oláh et al. [46] results. In this figure there is no real separation of the active and inactive branches and the Sun no longer looks like an outlier, raising questions over whether the Sun's activity cycle is unusual after all. 


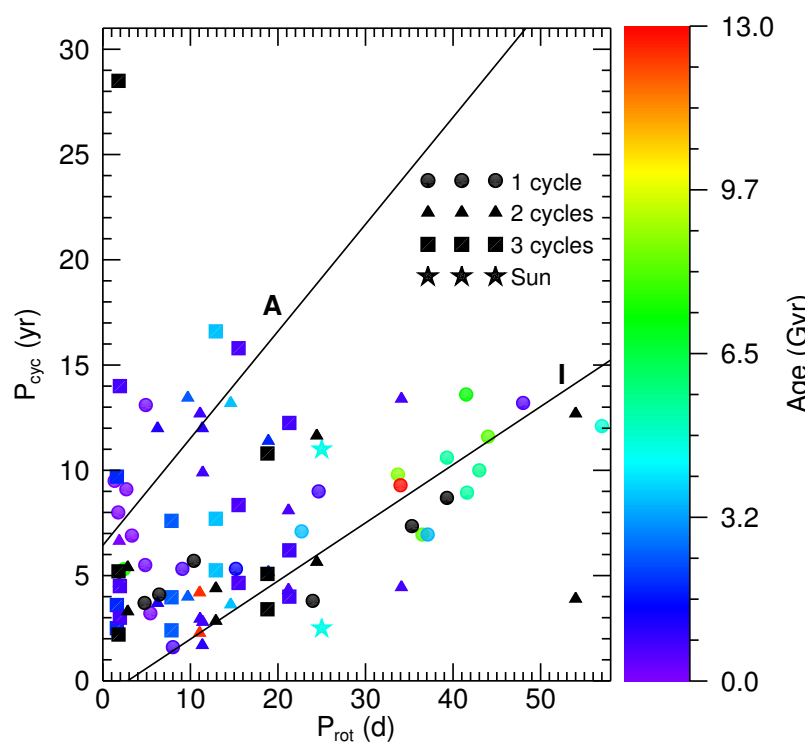

Figure 9: A Bohm-Vitense diagram constructed with the results of Oíah et al. [46]. Here symbols indicate the number of cycles Oláh et al. found in the Mount Wilson data.

\section{The seismology of stellar flares}

Seismology is now providing evidence for synergies between solar and stellar flares, which are associated with magnetic activity. Quasi-periodic pulsations (QPPs) are time variations in the intensity of the light associated with the flare. They can have periods from fractions of a second to tens of minutes and the properties of QPPs can provide information about the associated active region [see 47, 48, for recent reviews]. QPPs have also been observed in stellar flares since the 1970s [49]. However, observations of stellar QPP flares are sporadic and often serendipitously discovered. The potential of QPPs to link solar and stellar flares has already been recognised: Pugh et al. [50] detected multiple periodicities in a stellar flare light curve, the most plausible explanation for which is magnetohydrodynamic oscillations, analogous to those observed in solar flares. This not only implies a link between stellar flares and stellar magnetic activity but that the same physical processes are involved in solar and stellar flares.

Balona et al. [52] demonstrated that QPPs can be observed in Kepler flare light curves. Pugh et al. [51] searched the entire Kepler short cadence database for flares with evidence of QPPs and Figure 10 shows an example of one such flare. Out of over 200 flares detected in the Kepler short cadence database we found statistically significant evidence for QPPs in only 56 flares and just 11 of these showed QPPs persistent enough to fit a model to (as shown in Figure 10). Based on these 56 QPP flares we performed a statistical study, comparing QPP period to various stellar parameters, including radius, effective temperature, rotation rate, and $\log \mathrm{g}$ (see Figure 11). However, no significant correlations were found. This result is important as it implies that the QPP period is dependent upon the properties of the local active region from which it originated and not the global stellar parameters. This is exactly
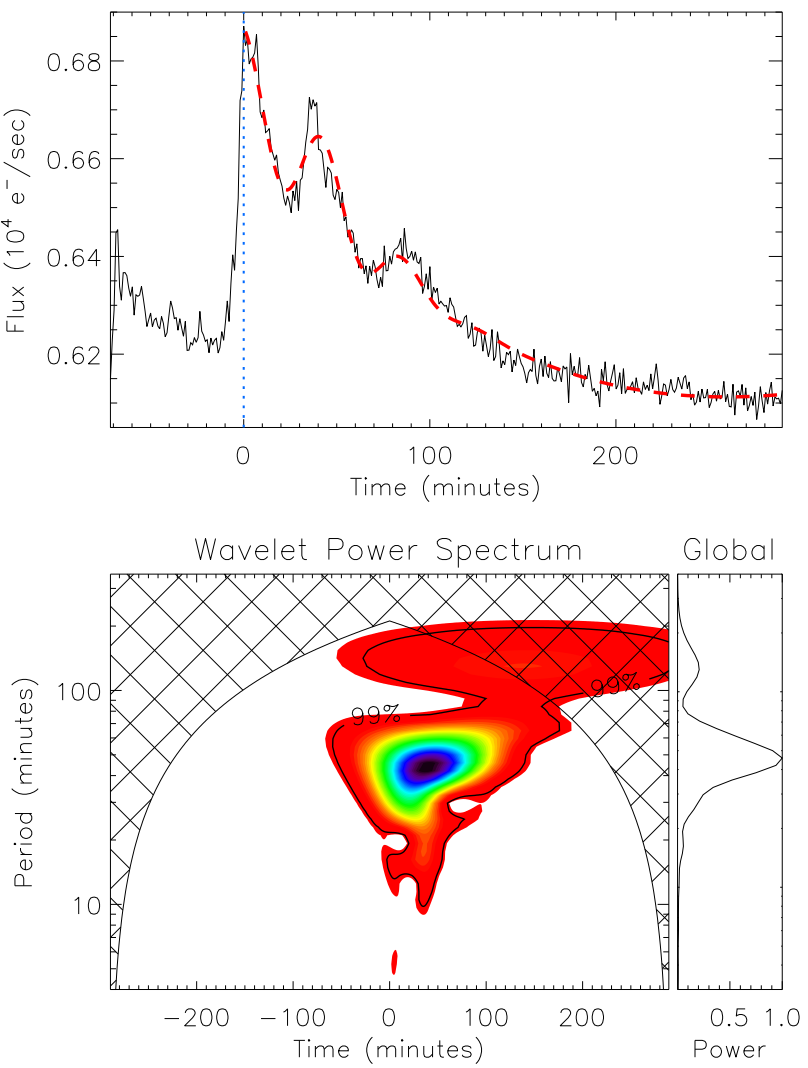

Figure 10: Top: Lightcurve of flare observed by Kepler on KIC12156549 (black). Overplotted in red is a fit to the data based on an exponential decay phase and a damped harmonic oscillator. Bottom: Wavelet spectrum of detrended light curve. Adapted from [51].

what one might expect based on solar observations, since the periods of QPPs observed on the Sun themselves extend over a wide range.

\section{Conclusions}

The Sun's activity cycle is far more complex than a simple $11 \mathrm{yr}$ (or even $22 \mathrm{yr}$ ) variation. Both the amplitudes and lengths change from one cycle to the next and numerous other timescales have been associated with it. Solar cycle variations in helioseismic parameters, including frequencies, damping rates and powers, are good proxies of solar activity. However, because the solar cycle is so complex observations of multiple cycles are required to gain insights into the chaotic nature of the Sun's dynamo.

We can search for similar variations in the oscillation parameters of other stars. However, to date only a few examples of asteroseismic activity cycles have been detected. Here we are limited by the length of data we have available and the fact that magnetic fields suppress the amplitudes of oscillations, meaning we can't perform asteroseismic studies on very magnetically active stars. We can, however, use seismology to study stellar flares. Although the field of stellar seismology is still very much in its infancy the first indications are that the physics of solar and stellar flares are the same. We can advance our understanding the 

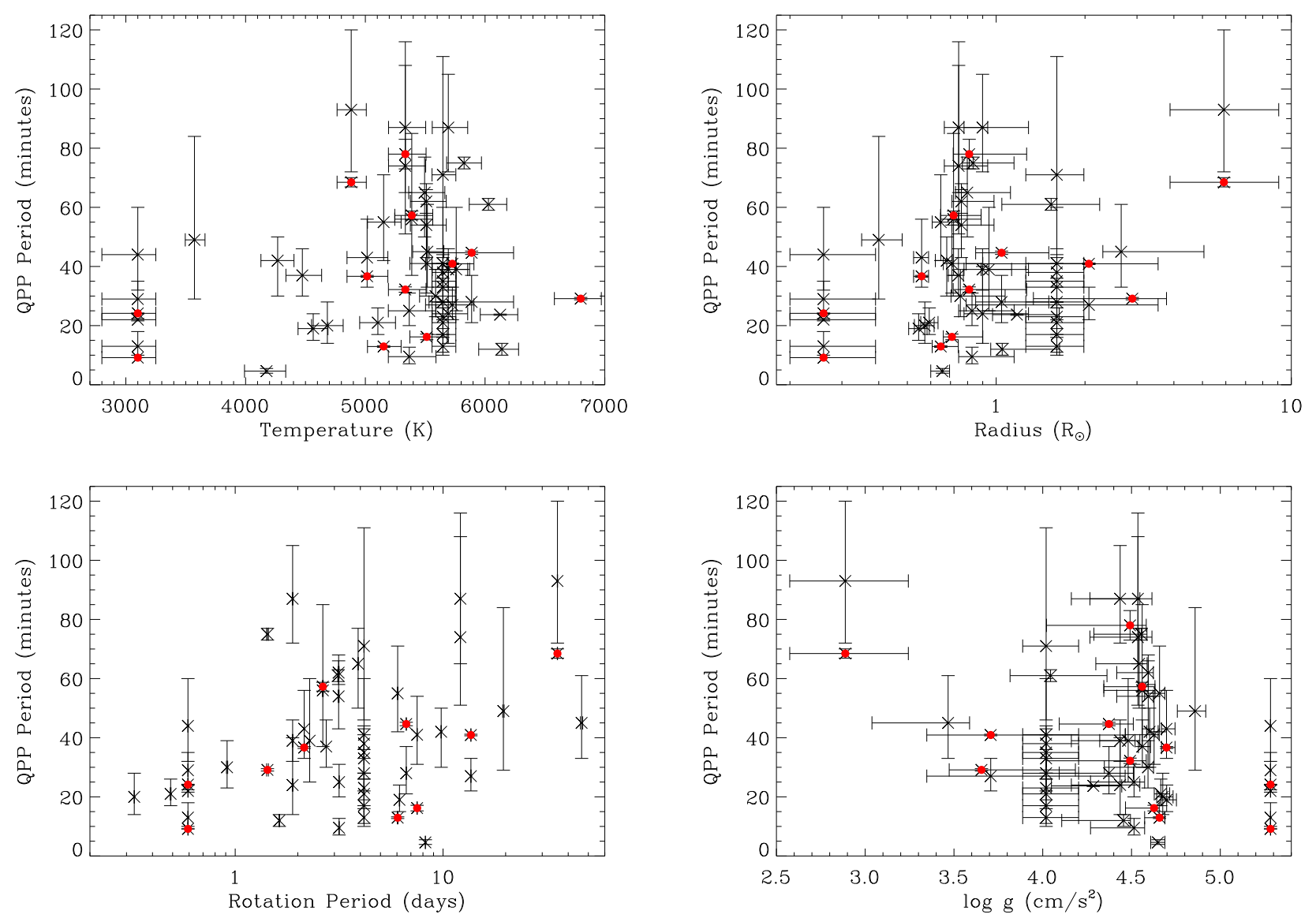

Figure 11: Scatter plots comparing the periodicity of the observed QPPs to various stellar parameters. Top left: QPP period as a function of effective temperature. Top right: QPP period as a function of stellar radius. Bottom left: QPP period as a function of stellar rotation period. Bottom right: QPP period as a function of log g. Black dots inditicate periodicities obtained from wavelet transforms. Red points indicate perioditicies obtained from fitting the decay light curve. Adapted from [51].

Sun's dynamo by comparing parameters important to dynamo processes as observed in the Sun and other stars and by placing the Sun in a stellar context.

\section{References}

[1] E. Rieger, G. Kanbach, C. Reppin, G.H. Share, D.J. Forrest, E.L. Chupp, Nature, 312, 623 (1984)

[2] G. Bazilevskaya, A.M. Broomhall, Y. Elsworth, V.M. Nakariakov, Space Science Reviews, 186, 359 (2014)

[3] W.J. Chaplin, Y. Elsworth, B.A. Miller, G.A. Verner, R. New, ApJ, 659, 1749 (2007)

[4] W.A. Dziembowski, P.R. Goode, ApJ, 625, 548 (2005), arXiv:astro-ph/0503266

[5] A.R.G. Santos, M.S. Cunha, P.P. Avelino, W.J. Chaplin, T.L. Campante, MNRAS, 461, 224 (2016), 1606.02133

[6] A.M. Broomhall, Sol. Phys., 292, 67 (2017), 1702.03149

[7] K.G. Libbrecht, M.F. Woodard, Nature, 345, 779 (1990)
[8] Y. Elsworth, R. Howe, G.R. Isaak, C.P. McLeod, B.A. Miller, R. New, C.C. Speake, S.J. Wheeler, ApJ, 434, 801 (1994)

[9] W.J. Chaplin, Y. Elsworth, G.R. Isaak, R. Lines, C.P. McLeod, B.A. Miller, R. New, MNRAS, 300, 1077 (1998)

[10] R. Howe, R. Komm, F. Hill, ApJ, 524, 1084 (1999)

[11] W.J. Chaplin, T. Appourchaux, Y. Elsworth, G.R. Isaak, R. New, MNRAS, 324, 910 (2001)

[12] S.J. Jiménez-Reyes, T. Corbard, P.L. Pallé, T. Roca Cortés, S. Tomczyk, A\&A, 379, 622 (2001), arXiv: astro-ph/0110364

[13] J. Christensen-Dalsgaard, G. Berthomieu, Theory of solar oscillations. (Solar Interior and Atmosphere, 1991), pp. 401-478

[14] D.O. Gough, Comments on Helioseismic Inference, in Progress of Seismology of the Sun and Stars, edited by Y. Osaki \& H. Shibahashi (1990), Vol. 367 of Lecture Notes in Physics, Berlin Springer Verlag, p. 283

[15] R. Howe, R.W. Komm, F. Hill, ApJ, 580, 1172 (2002) 
[16] A.M. Broomhall, P. Chatterjee, R. Howe, A.A. Norton, M.J. Thompson, Space Science Reviews, 186, 191 (2014), 1411.5941

[17] W.J. Chaplin, Y. Elsworth, G.R. Isaak, B.A. Miller, R. New, S. Thiery, P. Boumier, A.H. Gabriel, MNRAS, 343, 343 (2003)

[18] D.Y. Kolotkov, A.M. Broomhall, V.M. Nakariakov, MNRAS, 451, 4360 (2015)

[19] A.M. Broomhall, V.M. Nakariakov, Sol. Phys., 290, 3095 (2015), 1507.02854

[20] E.E. Benevolenskaya, ApJL, 509, L49 (1998), astro-ph/9810329

[21] T.V. Zaqarashvili, R. Oliver, J.L. Ballester, M. Carbonell, M.L. Khodachenko, H. Lammer, M. Leitzinger, P. Odert, A\&A, 532, A139 (2011), 1107.3395

[22] E. Covas, R. Tavakol, D. Moss, A\&A, 363, L13 (2000), astro-ph/0010491

[23] D. Moss, MNRAS, 306, 300 (1999)

[24] R. Simoniello, K. Jain, S.C. Tripathy, S. TurckChièze, C. Baldner, W. Finsterle, F. Hill, M. Roth, ApJ, 765, 100 (2013), 1210.6796

[25] W. Livingston, M.J. Penn, L. Svalgaard, ApJL, 757, L8 (2012)

[26] S. Basu, A.M. Broomhall, W.J. Chaplin, Y. Elsworth, ApJ, 758, 43 (2012), 1208.5493

[27] D. Salabert, R.A. García, S. Turck-Chièze, A\&A, 578, A137 (2015), 1502.07607

[28] A.M. Broomhall, C.E. Pugh, V.M. Nakariakov, Advances in Space Research 56, 2706 (2015)

[29] D.T. Woods, L.E. Cram, Sol. Phys., 69, 233 (1981)

[30] R. Howe, R.W. Komm, F. Hill, D.A. Haber, B.W. Hindman, ApJ, 608, 562 (2004)

[31] G. Houdek, W.J. Chaplin, T. Appourchaux, J. Christensen-Dalsgaard, W. Däppen, Y. Elsworth, D.O. Gough, G.R. Isaak, R. New, M.C. RabelloSoares, MNRAS, 327, 483 (2001)

[32] S.L. Baliunas, R.A. Donahue, W.H. Soon, J.H. Horne, J. Frazer, L. Woodard-Eklund, M. Bradford, L.M. Rao, O.C. Wilson, Q. Zhang et al., ApJ, 438, 269 (1995)

[33] S.L. Baliunas, R.A. Donahue, W. Soon, G.W. Henry, Activity Cycles in Lower Main Sequence and POST Main Sequence Stars: The HK Project, in Cool Stars, Stellar Systems, and the Sun, edited by R.A. Donahue, J.A. Bookbinder (1998), Vol. 154 of Astronom- ical Society of the Pacific Conference Series, p. 153

[34] R.A. García, S. Mathur, D. Salabert, J. Ballot, C. Régulo, T.S. Metcalfe, A. Baglin, Science 329, 1032 (2010), 1008.4399

[35] D. Salabert, C. Régulo, J. Ballot, R.A. García, S. Mathur, A\&A, 530, A127 (2011), 1104.5654

[36] W.J. Chaplin, Y. Elsworth, G. Houdek, R. New, MNRAS, 377, 17 (2007)

[37] T.S. Metcalfe, W.A. Dziembowski, P.G. Judge, M. Snow, MNRAS, 379, L16 (2007), 0704 . 1606

[38] D. Salabert, C. Régulo, R.A. García, P.G. Beck, J. Ballot, O.L. Creevey, F. Pérez Hernández, J.D. do Nascimento, Jr., E. Corsaro, R. Egeland et al., A\&A, 589, A118 (2016), 1603.00655

[39] C. Régulo, R.A. García, J. Ballot, A\&A, 589, A103 (2016), 1603.04673

[40] R. Kiefer, A. Schad, G. Davies, M. Roth, A\&A, 598, A77 (2017), 1611.02029

[41] E. Böhm-Vitense, ApJ, 657, 486 (2007)

[42] N.J. Wright, J.J. Drake, E.E. Mamajek, G.W. Henry, ApJ, 743, 48 (2011), 1109. 4634

[43] W.J. Chaplin, T.R. Bedding, A. Bonanno, A.M. Broomhall, R.A. García, S. Hekker, D. Huber, G.A. Verner, S. Basu, Y. Elsworth et al., ApJL, 732, L5 (2011), 1103.5570

[44] J.L. van Saders, T. Ceillier, T.S. Metcalfe, V. Silva Aguirre, M.H. Pinsonneault, R.A. García, S. Mathur, G.R. Davies, Nature, 529, 181 (2016), 1601.02631

[45] T.S. Metcalfe, R. Egeland, J. van Saders, ApJL, 826, L2 (2016), 1606.01926

[46] K. Oláh, Z. Kővári, K. Petrovay, W. Soon, S. Baliunas, Z. Kolláth, K. Vida, A\&A, 590, A133 (2016), 1604.06701

[47] V.M. Nakariakov, V.F. Melnikov, Space Science Reviews, 149, 119 (2009)

[48] T. Van Doorsselaere, E.G. Kupriyanova, D. Yuan, Sol. Phys., 291, 3143 (2016), 1609.02689

[49] M. Rodono, A\&A, 32, 337 (1974)

[50] C.E. Pugh, V.M. Nakariakov, A.M. Broomhall, ApJL, 813, L5 (2015), 1510.03613

[51] C.E. Pugh, D.J. Armstrong, V.M. Nakariakov, A.M. Broomhall, MNRAS, 459, 3659 (2016), 1604.03018

[52] L.A. Balona, A.M. Broomhall, A. Kosovichev, V.M. Nakariakov, C.E. Pugh, T. Van Doorsselaere, MNRAS, 450, 956 (2015), 1504 . 01491 